\title{
BIBLIOGRAPHY
}

1. H. Seifert and W. Thelfall, Lehrbuch der Topologie, Leipzig and Berlin, 1934.

2. A. M. Peiser, Covering mappings, Duke Math. J. vol. 10 (1943) pp. 305-307.

3. K. Borsuk, Sur les retractes, Fund. Math. vol. 17 (1931) pp. 152-170.

4. C. W. Saalfrank, Retraction properties for normal Hausdorff spaces, Fund. Math. vol. 36 (1949) pp. 93-108.

RUTGERS UNIVERSITY AND

LAFA YetTE COLLEGE

\section{DENSE IMBEDDING OF TOPOLOGICAL GROUPS}

\section{MORIKUNI GOTO 1}

The present article originated from the problem of determining when a continuous representation of a Lie group into another is open. Important cases of the problem have been discussed by A. Malcev² and the author, ${ }^{8}$ and some of them have been extended to the case of more general groups by the author and H. Yamabe.4

Recently W. T. van Est obtained new results concerning the same problem on Lie groups. ${ }^{5}$ Here I shall give an extension of his essential result to a more general case in a simpler way.

Let $G$ be a locally compact connected group and let $A(G)$ be the group of all continuous automorphisms of $G$. Let $A(G)$ be topologized by the notion of uniform convergence in the wider sense.

Now let $I(G)$ be the subgroup of $A(G)$ composed of all inner automorphisms of $G$. We shall call $G$ a (CA) group ${ }^{6}$ if $I(G)$ is a closed subgroup of $A(G)$.

LeмmA. ${ }^{7}$ Let $G$ be a locally compact connected and locally connected group, and $H$ a locally compact group. If $\phi$ is a continuous isomorphism which maps $G$ in an everywhere dense subgroup in $H$, then the following propositions hold:

(1) $\phi(G)$ is an invariant subgroup of $H$.

(2) Let $h$ be an element of $H$. Let us consider the automorphism $\sigma_{h}(x)$ defined by $\sigma_{h} x=\phi^{-1}\left(h^{-1} \phi(x)\right) h$ for $x \in G$. Then $\sigma_{h}(x)$ is a continuous

Received by the editors October $6,1952$.

1 The author's name will be hereafter spelled "Goto" instead of "Goto" which has been used thus far.

${ }^{2}$ Malcev [4] in the bibliography.

${ }^{3}$ Goto [1].

4 Goto and Yamabe [3]. See also [2].

${ }^{5}$ van Est [5], where he solved a prize problem (Wiskundig Genootshap Amsterdam, 1950), which had already been established in [1] and generalized in [3], independently of the author.

- The notion of a (CA) group is a generalization of van Est's (CA) Lie group.

${ }^{7}$ See $[3]$ and [2]. 
automorphism of $G$.

(3) The correspondence $h \rightarrow \sigma_{h}$ defines a continuous homomorphism from $H$ into $A(G)$.

From this lemma we infer the following theorem.

THEOREM. ${ }^{8}$ Let $G$ be a locally compact connected and locally connected (CA) group, and $H$ a locally compact group. Suppose that the center $Z$ of $G$ is compact. If there exists a continuous isomorphism $\phi$ mapping $G$ into $H$, then the image $\phi(G)$ is a closed subgroup of $H$.

Proof. Clearly we need consider only the case when $H$ coincides with the closure $\operatorname{Cl}(\phi(G))$ of $\phi(G)$. In such a case we get a continuous homomorphism $h \rightarrow \sigma_{h}$ from $H$ into $A(G)$ by virtue of the above lemma, and $I(G)$ corresponds to $\phi(G)$ under this homomorphism: $\sigma \phi(G)=I(G)$. Now since $\sigma$ is continuous we get $\sigma \mathrm{Cl}(\phi(G)) \subseteq \mathrm{Cl}(I(G))$ and thus $\sigma(H) \subseteq I(G) .{ }^{9}$ The above relation means that for every $h$ in $H$ there exists an element $g$ of $G$ such that

$$
\phi(g)^{-1} x \phi(g)=h^{-1} x h \quad \text { for all } x \in \phi(G) .
$$

Then $h \phi(g)^{-1}$ commutes with every element of $\phi(G)$, whence $h \phi(g)^{-1}$ is contained in the center $A$ of $H$. Thus we have

$$
H=\phi(G) A .
$$

Since $Z$ is compact, $\phi(Z)$ is also compact, and the relation $\phi(G) \cap A$ $=\phi(Z)$ is obvious. Hence we have algebraically

$$
H / \phi(Z)=\phi(G) / \phi(Z) \times A / \phi(Z)
$$

where $X$ means the direct product of abstract groups.

Let us now consider the topological direct product group

$$
L=G / Z \times A / \phi(Z)
$$

of locally compact groups $G / Z$ and $A / \phi(Z)$. It is easy to show that $L$ can be covered by countable compact sets. Hence the continuous isomorphism from $L$ onto $H / \phi(Z)$, obtained by extending the mapping $\phi$ and the identity mapping of $A / \phi(Z)$, is necessarily open. Therefore $\phi(G) / \phi(Z)$ is closed in $H / \phi(Z)$, whence $\phi(G)$ is closed in $H$; this completes the proof.

Corollary. ${ }^{10}$ Let $G$ be a local Lie group. Then $G$ always generates $a$

8 This theorem includes the essential theorems in both [3] and [5].

- The following argument is entirely the same as one in [3].

${ }^{10}$ Our theorem is superfluous for obtaining this corollary, which can be proved by the theorem of van Est only. 
closed subgroup whenever $G$ is imbedded in a Lie group as a local subgroup if and only if the following two conditions are satisfied:

(1) The adjoint group of $G$ is a closed linear group.

(2) The center of the simply-connected group corresponding to $G$ is a finite group.

Proof. Necessity: (2) is already known. ${ }^{11}$ Then as the adjoint group of $G$ is locally isomorphic with $G$, it must be closed in the general linear group.

The sufficiency follows readily from the theorem.

\section{Bibliography}

1. M. Goto, Faithful representations of Lie groups I, Mathematica Japonicae vol. 1 (1948).

2. - On local Lie groups in a locally compact group, Ann. of Math. vol. 54 (1951).

3. M. Goto and H. Yamabe, On continuous isomorphisms of topological groups, Nagoya Math. J. vol. 1 (1950).

4. A. Malcev, On the theory of Lie groups in the large, Rec. Math. (Mat. Sbornik) vol. 16 (1945).

5. W. T. van Est, Dense imbeddings of Lie groups, Neder. Akad. Wetensch. vol. 54 (1951).

The Institute for Advanced Study

11 See [1] and [5]. 